\title{
Magnetocaloric effect and spin-strain coupling in the spin-nematic state of $\mathrm{LiCuVO}_{4}$
}

\author{
M. Gen, ${ }^{1}$ T. Nomura, ${ }^{2}$ D. I. Gorbunov, ${ }^{2}$ S. Yasin, ${ }^{2}$ P. T. Cong, ${ }^{2}$ C. Dong,,${ }^{1,3}$ Y. Kohama, ${ }^{1}$ E. L. Green,,${ }^{2,}$ J. M. Law, ${ }^{2}$ \\ M. S. Henriques, ${ }^{4}$ J. Wosnitza, ${ }^{2,5}$ A. A. Zvyagin, ${ }^{6,7,8}$ V. O. Cheranovskii, ${ }^{8}$ R. K. Kremer, ${ }^{9}$ and S. Zherlitsyn ${ }^{2}{ }^{2}$ \\ ${ }^{1}$ Institute for Solid State Physics, University of Tokyo, Kashiwa, Chiba 277-8581, Japan \\ ${ }^{2}$ Hochfeld-Magnetlabor Dresden (HLD-EMFL) and Würzburg-Dresden Cluster of Excellence ct.qmat, \\ Helmholtz-Zentrum Dresden-Rossendorf, 01328 Dresden, Germany \\ ${ }^{3}$ Wuhan National High Magnetic Field Center and School of Physics, Huazhong University of Science and Technology, Wuhan 430074, China \\ ${ }^{4}$ Institute of Physics, Czech Academy of Sciences, Na Slovance 2, 18221 Prague, Czech Republic \\ ${ }^{5}$ Institut für Festkörper- und Materialphysik, Technische Universität Dresden, 01062 Dresden, Germany \\ ${ }^{6}$ Max-Planck-Institut für Physik komplexer Systeme, 01187 Dresden, Germany \\ ${ }^{7}$ B. I. Verkin Institute for Low Temperature Physics and Engineering of the National Academy of Sciences of Ukraine, Kharkiv 61103, Ukraine \\ ${ }^{8}$ V. N. Karazin Kharkiv National University, 4 Svoboda Square, Kharkiv 61022, Ukraine \\ ${ }^{9}$ Max-Planck-Institut für Festkörperforschung, 70569 Stuttgart, Germany
}

(Received 28 January 2019; revised manuscript received 20 September 2019; published 31 October 2019)

\begin{abstract}
We report on the magnetocaloric effect and ultrasound studies of the frustrated quasi-one-dimensional spin-1/2 compound $\mathrm{LiCuVO}_{4}$, evidencing a spin-nematic state. The magnetic Grüneisen parameter diverges at the transition to the spin-nematic phase, $\mu_{0} H_{\mathrm{c} 3} \approx 40 \mathrm{~T}$, showing quantum criticality accompanied by entropy accumulation. The observed high-field anomalies in the acoustic properties clearly evidence a strong involvement of the lattice in the spin dynamics. The theoretical approach, based on exchange-striction coupling with dipolar and quadrupolar contributions, suggests that the spin-dipole-strain and quadrupole-strain interactions govern the spin-nematicity in $\mathrm{LiCuVO}_{4}$.
\end{abstract}

DOI: 10.1103/PhysRevResearch.1.033065

\section{INTRODUCTION}

Spin-nematic phases in which the spin-rotational symmetry is broken whereas translational and time-reversal symmetry are preserved constitute the magnetic analog of nematic liquid crystals [1]. The spin-nematic state is characterized by a spin-quadrupolar order. However, since there is no simple electromagnetic field coupling directly to quadrupolar order the experimental techniques applied to characterize the spinnematic state, so far, have been indirect and rather insensitive to the nematic order.

There is currently an active search for model magnetic systems which support spin-nematic phases. The most promising system currently considered to establish a spin-nematic phase is the compound $\mathrm{LiCuVO}_{4} \cdot \mathrm{LiCuVO}_{4}$ contains $\mathrm{Cu}^{2+}$ linear spin-1/2 chains with nearest-neighbor $(\mathrm{NN})$ ferromagnetic (FM) $\left(J_{1}=-1.6 \mathrm{meV}\right)$ and next-nearest-neighbor (NNN) antiferromagnetic (AFM) $\left(J_{2}=3.8 \mathrm{meV}\right)$ exchange coupling equivalent to a geometrically frustrated zigzag arrangement running along the $b$ direction [2,3]. Weak interchain coupling $J=-0.4 \mathrm{meV}$ leads to long-range AFM order below $T_{\mathrm{N}} \approx$

\footnotetext{
*Present address: National High Magnetic Field Laboratory, Florida State University, Tallahassee, Florida 32310, USA.

Published by the American Physical Society under the terms of the Creative Commons Attribution 4.0 International license. Further distribution of this work must maintain attribution to the author(s) and the published article's title, journal citation, and DOI.
}

$2.3 \mathrm{~K}$ with an incommensurate planar helicoidal spin structure with the magnetic moments lying in the $a b$ plane [4-6]. In magnetic fields above $\mu_{0} H_{\mathrm{c} 2} \approx 7.5 \mathrm{~T}$ applied along $c$ perpendicular to the spin helices, an incommensurate, collinear spindensity wave (SDW) phase is stabilized [7-9]. Zhitomirsky and Tsunetsugu have proposed that in $\mathrm{LiCuVO}_{4}$ close to magnetic saturation the two-magnon bound state has a lower energy than the one-magnon excitation [10]. Two-magnon condensation [11-14] aggregates the nematic state which bears some similarity with a "bosonic superconductor" [15].

High-field magnetization and ${ }^{51} \mathrm{~V}$ nuclear magnetic resonance results obtained just below the spin-saturated state have recently been reported as evidence for a spin-nematic phase in $\mathrm{LiCuVO}_{4}[16,17]$. From the currently available high-field magnetization results the spin-nematic phase in $\mathrm{LiCuVO}_{4}$ has been suggested to exist from $\mu_{0} H_{\mathrm{c} 3}$ of about $40 \mathrm{~T}$ for $H \| c$ and $47 \mathrm{~T}$ for $H \| a$ or $b$ to the saturation field $\mu_{0} H_{\text {sat }} \approx 44.5$ and $52 \mathrm{~T}$, respectively, for the $H \| c$ and $H \| a$ or $b$ directions $[16,18]$.

In this paper we report on an investigation of the spinnematic state in $\mathrm{LiCuVO}_{4}$ by the pulsed high-field magnetocaloric effect (MCE) and ultrasound measurements. The MCE, i.e., the temperature change induced by a magnetic field change, provides information about the magnetic entropy balance in a magnetic system [19]. The magnetic Grüneisen parameter derived from the MCE, $\Gamma_{H} \equiv(1 / T)\left[d T / d\left(\mu_{0} H\right)\right]$, has been shown to constitute an excellent tool to identify putative quantum-critical points [20]. Ultrasound propagation is particularly sensitive to quadrupolar degrees of freedom [21] 
and therefore ideally suited to study a nematic phase. In addition, spin-strain interactions known to be important in the physics of low-dimensional [21-23] and frustrated spin systems [24-27] can favorably be studied by the ultrasound technique. Our experiments reveal a divergence of the magnetic Grüneisen parameter accompanied by entropy accumulation at the phase transition to the spin-nematic state in $\mathrm{LiCuVO}_{4}$. The ultrasound attenuation results show contributions from the spin-quadrupolar and spin-dipolar degrees of freedom in the spin-nematic state of $\mathrm{LiCuVO}_{4}$.

\section{EXPERIMENTAL DETAILS}

A high-quality $\mathrm{LiCuVO}_{4}$ sample from the same batch used in Refs. [17,28] was studied. $\mathrm{LiCuVO}_{4}$ has an orthorhombically distorted spinel structure with space group Imma [29-31]. To determine the crystallographic orientation of our sample, we used a four-circle x-ray diffractometer, equipped with a Mo x-ray tube $\left[\lambda\left(\right.\right.$ Mo- $\left.\left.K_{\alpha}\right)=0.71073 \AA\right]$. The lattice parameters were found to be $a=5.644(3) \AA$, $b=5.842(3) \AA$, and $c=8.738(5) \AA$, in agreement with previously published results [32]. The same single crystal $(1.9 \times$ $2.0 \times 0.65 \mathrm{~mm}^{3}$ ) was studied in the MCE and ultrasound experiments. The MCE was measured in pulsed magnetic fields up to $50 \mathrm{~T}$ by using a $\mathrm{Au}_{16} \mathrm{Ge}_{84}$ thin-film thermometer [33]. In order to ensure adiabatic conditions, the sample was thermally isolated in vacuum. The sound-velocity and sound-attenuation measurements were performed employing a phase-sensitive detection technique [21]. The shape of the sample allowed us to investigate two quasitransverse acoustic modes, $c_{\mathrm{t}}$ and $c_{\mathrm{t}^{\prime}}$, with wave vector $\mathbf{k}$ in the $a b$ plane $(\mathbf{k} \|[110])$ and polarization $\mathbf{u}$ in $(\mathbf{u} \|[1 \overline{1} 0])$ and perpendicular to the $a b$ plane (u\|[001]), respectively, and one longitudinal acoustic mode, $c_{33}(\mathbf{k}\|\mathbf{u}\|[001])$, as well. The elastic constants $c_{i j}=$ $\rho v^{2}$ are proportional to the square of the corresponding sound velocities, $v$, where $\rho$ is the mass density. Note that the $a$ and $b$ lattice parameters have only modestly different values; i.e., the crystal structure is metrically almost tetragonal. This justifies using the quasitransverse instead of pure transverse acoustic modes. The acoustic $c_{\mathrm{t}}$ mode corresponds to the pure $\left(c_{11}-c_{12}\right) / 2$ mode in tetragonal notation, if $a$ and $b$ would be exactly equal. The other transverse acoustic mode, $c_{t^{\prime}}$, would be the pure $c_{44}$ mode in this case. The magnetic field was always applied along [001] since $\mu_{0} H_{\mathrm{c} 3}$ and $\mu_{0} H_{\mathrm{sat}}$ had the lowest values for this crystallographic direction.

\section{RESULTS AND DISCUSSION}

\section{A. Magnetocaloric effect}

The temperature change of a $\mathrm{LiCuVO}_{4}$ crystal initiated by ramping the magnetic field $(H \| c)$ up and down (MCE) is displayed in Fig. 1(a). In the region $T>4 \mathrm{~K}$ and $\mu_{0} H>40 \mathrm{~T}$ the field-induced temperature changes are reversible, assuring adiabatic conditions and representing isentropic curves in this regime. For all measured temperatures, $T(H)$ exhibits a marked dip in the vicinity of $\mu_{0} H_{\mathrm{c} 3} \approx 40 \mathrm{~T}$. The dip in $T(H)$ indicates maximum accumulation of magnetic entropy,
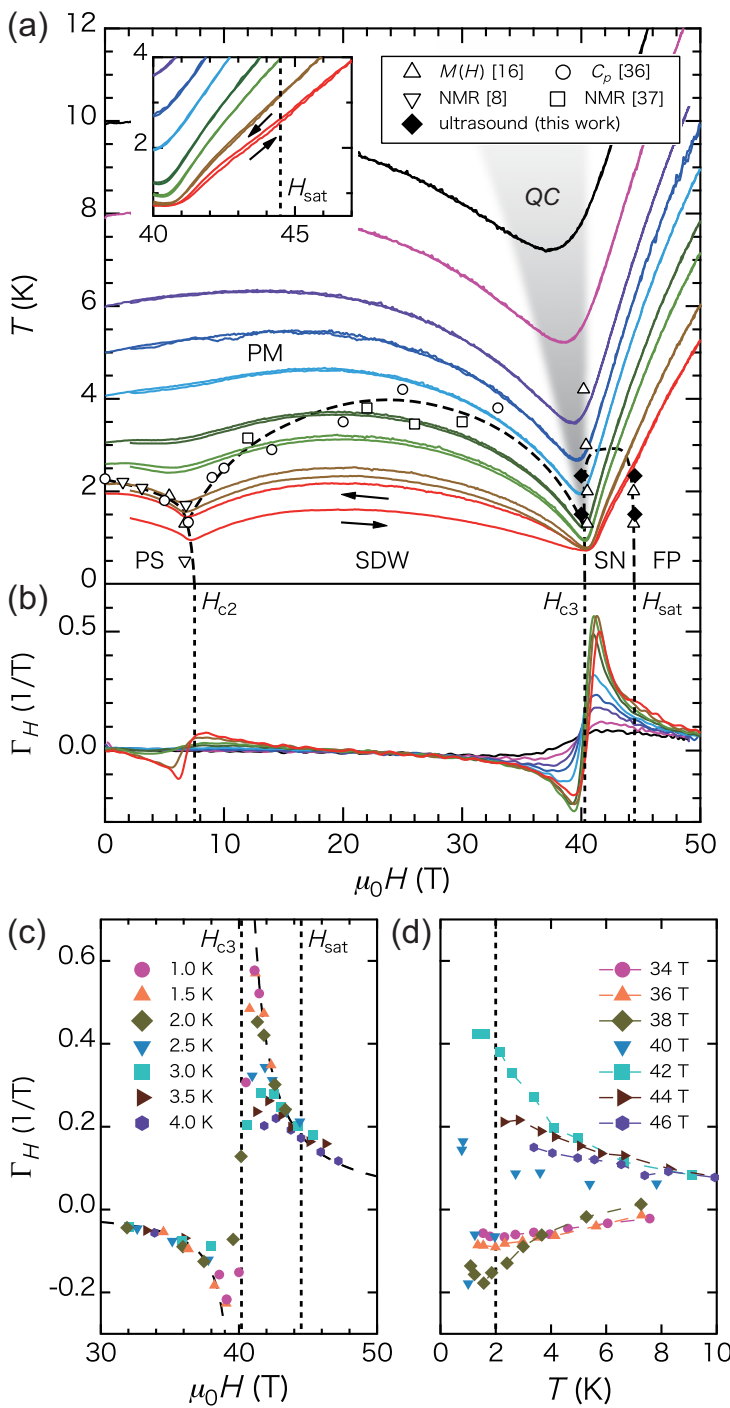

FIG. 1. (a) Magnetocaloric effect in $\mathrm{LiCuVO}_{4}$ measured at various initial temperatures in pulsed magnetic fields $(H \| c)$. The inset shows an enlarged view near the saturation field. The symbols denote phase transitions extracted from previous studies (Refs. [8,16,36,37]) and our ultrasound results. The dashed lines are guides to the eye and separate the paramagnetic (PM), the incommensurate planar spiral (PS), the collinear SDW, the spin-nematic (SN), and the fully polarized (FP) phases. Gray shadow shows the quantumcritical (QC) regime around $\mu_{0} H_{\mathrm{c} 3}$ as evidenced by the minimum in the MCE. (b) Magnetic Grüneisen parameter, $\Gamma_{H}$, obtained along the isentropic MCE curves for the down sweeps. The color of each curve corresponds to that of Fig. 1(a). (c) $\Gamma_{H}$ versus magnetic field at several constant temperatures are plotted. The data follow the critical divergence $\Gamma_{H} \propto \mu_{0}^{-1}\left(H-H_{\mathrm{c} 3}\right)^{-1}$ (dashed lines). (d) $\Gamma_{H}$ versus temperature at some selected magnetic fields.

evidencing that strong spin fluctuations are present at temperatures much higher than $T_{\mathrm{N}}$. Notable changes in the field dependence of $T(H)$ occur when the initial temperature is chosen below $T_{\mathrm{N}}$ : (i) the MCE becomes hysteretic, especially below $H_{\mathrm{c} 3}$; (ii) the dip at $\mu_{0} H_{\mathrm{c} 3} \approx 40 \mathrm{~T}$ sharpens; and (iii) a kink appears in the low-field region around $\mu_{0} H_{\mathrm{c} 2} \approx 7.5 \mathrm{~T}$. 
This kink is seen at the field where the transition from the incommensurate planar spiral to the collinear SDW takes place [34]. The hysteresis, most pronounced below $\mu_{0} H_{\mathrm{c} 3}$, is attributed to the first-order nature of the transition from the SDW to a spin-nematic phase proposed theoretically $[10,35]$. A closer inspection of $T(H)$ for the lowest initial temperature reveals a tiny change of slope accompanied by a hysteresis near $\mu_{0} H_{\text {sat }} \approx 44.5 \mathrm{~T}$ [see inset of Fig. 1(a)]. The hysteresis is, however, partially masked by the large MCE heating above $H_{\mathrm{c} 3}$.

The magnetic Grüneisen parameter $\Gamma_{H}$ [Fig. 1(b)] exhibits a singularity at $H_{\mathrm{c} 3}$ with a sign change evidencing quantum criticality [20]. Figures 1(c) and 1(d) show the isothermal and isomagnetic Grüneisen parameter around $H_{\mathrm{c} 3}$ versus magnetic field and temperature, respectively. The critical divergence of $\Gamma_{H}$ at $\mu_{0} H_{\mathrm{c} 3} \approx 40 \mathrm{~T}$ is well described by a power law $\mu_{0}^{-1} G_{r}\left(H-H_{\mathrm{c} 3}\right)^{-1}$ with a prefactor $G_{r}=0.80 \pm 0.05$ for $H>H_{\mathrm{c} 3}$ and $G_{r}=0.30 \pm 0.03$ for $H<H_{\mathrm{c} 3}$, respectively [dashed lines in Fig. 1(c)]. Divergent behavior of the magnetic Grüneisen parameter has similarly been observed in other 1D spin-chain systems [19,38,39]. The divergence of $\Gamma_{H}$ appears to saturate below $\sim 2 \mathrm{~K}$ [Fig. 1(d)], indicating that quantum fluctuations become weaker in the hysteretic state between the SDW and the spin-nematic phases. An analogous feature was observed for the $S=1 / 2$ square-lattice $J_{1}-J_{2}$ antiferromagnet $\mathrm{BaCdVO}\left(\mathrm{PO}_{4}\right)_{2}$, which has also been proposed to develop a spin-nematic phase just below the saturated state [40]. The analysis of the MCE and magnetic Grüneisen parameter provides strong evidence that at $\mu_{0} H_{\mathrm{c} 3} \approx 40 \mathrm{~T}$ for temperatures below $\sim 2 \mathrm{~K}$ a first-order transition to a new phase takes place which we associate with the spin-nematic phase proposed theoretically and also seen in high-field NMR experiments $[10,17,35]$.

\section{B. Magnetoacoustics}

A first-order transition at $\mu_{0} H_{\mathrm{c} 3} \approx 40 \mathrm{~T}$ in $\mathrm{LiCuVO}_{4}$ is also confirmed by our ultrasound results presented in Fig. 2. At $1.5 \mathrm{~K}$, the sound velocities of the transverse $c_{\mathrm{t}}$ and $c_{\mathrm{t}^{\prime}}$ modes are continuously increasing with magnetic field up to the transition to the spin-nematic state. At $\mu_{0} H_{\mathrm{c} 3} \approx 40 \mathrm{~T}$ maxima in the sound velocity and attenuation appear, followed by a steep decrease. A second peak appears when entering the saturated state at $\mu_{0} H_{\text {sat }} \approx 44.5 \mathrm{~T}$. Above $\mu_{0} H_{\text {sat }}$ the sound velocity remains at a constant level. Between about 22 and $50 \mathrm{~T}$ at $T=1.5 \mathrm{~K}$, we find a clear hysteresis in the acoustic properties. The appearance of the hysteresis at $\sim 22 \mathrm{~T}$ may indicate a crossover from the SDW to a spinnematic Tomonaga-Luttinger liquid expected for a $1 \mathrm{D} J_{1}-J_{2}$ spin chain as discussed in Ref. [41]. Remarkably, a hysteresis is seen at $2.3 \mathrm{~K}$ and even at $4.2 \mathrm{~K}$. The longitudinal $c_{33}$ mode does not exhibit any anomalous behavior within experimental resolution [42]. Note that only transverse acoustic modes can effectively couple to quadrupolar degrees of freedom [21]. In summary, our measurements of the ultrasound propagation in $\mathrm{LiCuVO}_{4}$ evidence hysteretic behavior of the acoustic properties and distinct maxima in the velocity and attenuation of the transverse acoustic modes when entering the spin-nematic state.

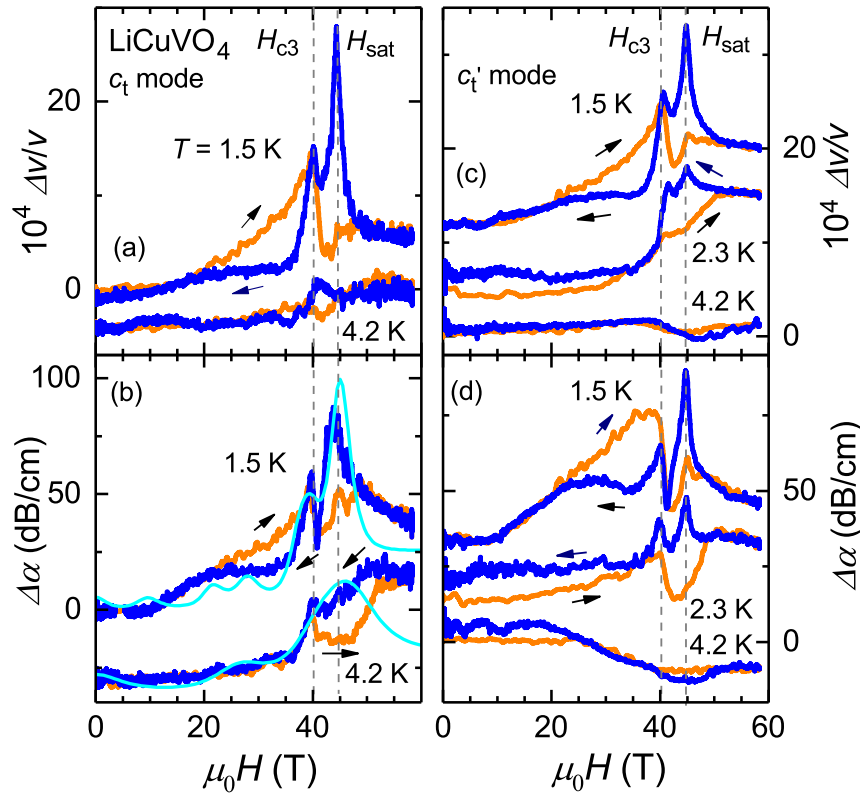

FIG. 2. Sound-velocity and sound-attenuation change for the transverse acoustic (a), (b) $c_{\mathrm{t}}$ and (c), (d) $c_{\mathrm{t}^{\prime}}$ mode, respectively, measured in pulsed magnetic fields at 1.5, 2.3, and 4.2 K. Results for up and down field sweeps are shown. The ultrasound frequency was $110 \mathrm{MHz}$. The spin-nematic state is realized between $H_{\mathrm{c} 3}$ and the saturation field, $H_{\text {sat }}$. The cyan solid lines in (b) depict the calculated magnetic field dependence of the sound attenuation of the $c_{\mathrm{t}}$ mode of $\mathrm{LiCuVO}_{4}$ at $T=1.5$ and $4.2 \mathrm{~K}$ (see text for details).

Figure 1(a) shows our MCE results together with the $H-T$ phase diagram obtained from previous NMR $[8,16]$, magnetization [36], specific-heat [37], and our present ultrasound experiments. Obviously, our data are consistent with the earlier results. In this work, we concentrate on the high-field part of the phase diagram, specifically on the spin-nematic state. The low-field phase transitions, such as from the paramagnetic to the incommensurate planar spiral or SDW states, are beyond the scope of this work.

Now, we consider spin-strain effects on the ultrasound properties at zero magnetic field, where the quadrupolar degrees of freedom are irrelevant. Figure 3 shows the temperature dependence of the sound velocity for the acoustic $c_{\mathrm{t}}$ mode, measured at two selected frequencies. A striking feature is the strong softening of the $c_{\mathrm{t}}$ mode with decreasing temperature starting already at $\sim 75 \mathrm{~K}$. Two minima appear below $30 \mathrm{~K}$. Their frequency dependence hints at relaxation processes due to the spin dynamics of the short-range correlated regime, which was suggested from NMR studies on $\mathrm{LiCuVO}_{4}$ to occur below $15 \mathrm{~K}$ down to $T_{\mathrm{N}}$ [7]. Due to the large background softening of the $c_{\mathrm{t}}$ mode, it is challenging to extract the corresponding magnetic relaxation time and characterize the relaxation processes in more detail. Note that the short-range order maximum in the magnetic susceptibility associated with the appearance of 1D AFM correlations occurs at about $28 \mathrm{~K}$ [3]. In contrast to the $c_{\mathrm{t}}$ mode, the longitudinal $c_{33}$ mode exhibits only a slight additional stiffening below $10 \mathrm{~K}$ on top of the usual velocity increase due to the anharmonicity of the ionic potential [43]. 


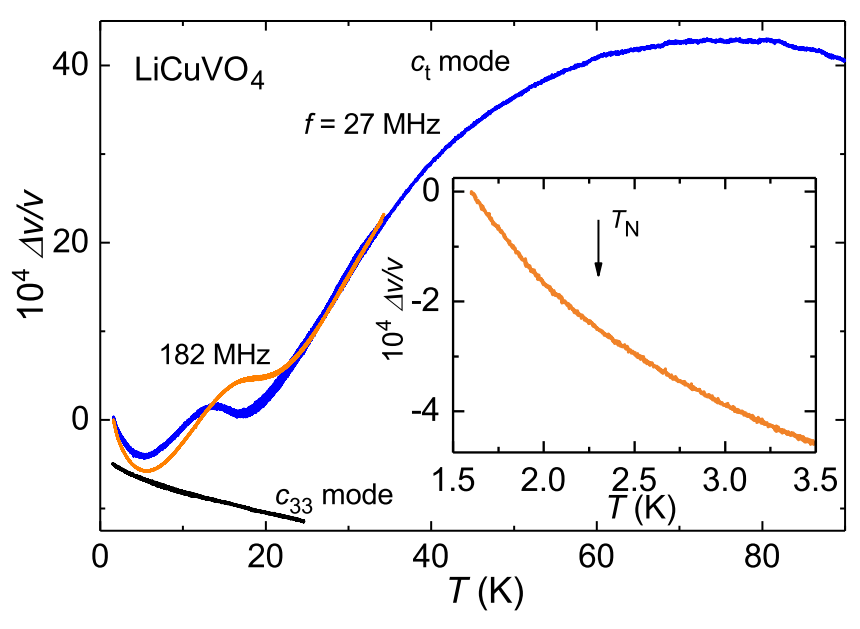

FIG. 3. Temperature dependence of the relative change of the sound velocity $\Delta v / v$ for the acoustic $c_{\mathrm{t}}$ and $c_{33}$ modes in $\mathrm{LiCuVO}_{4}$. For the $c_{\mathrm{t}}$ mode the results obtained at 27 (blue line) and $182 \mathrm{MHz}$ (orange line) are shown. In the case of the $c_{33}$ mode (black line) the ultrasound frequency was $52 \mathrm{MHz}$. The inset shows the sound velocity for the $c_{\mathrm{t}}$ mode in the vicinity of the AFM ordering.

Evidently, spin-strain coupling is much stronger for the acoustic $c_{\mathrm{t}}$ mode inducing the large softening of $c_{\mathrm{t}}$ due to enhanced spin-spin correlations appearing already in the paramagnetic state far above $T_{\mathrm{N}}$. Remarkably, a large part of the $c_{\mathrm{t}}$ mode stiffness is recovered in the spin-nematic state, as seen by the increase of the sound velocity [Fig. 2(a)]. However, the sound-velocity increase in the spin-nematic phase cannot be explained based on temperature changes due to the MCE effect. Note that no clear sound velocity anomaly is detected in the vicinity of $T_{\mathrm{N}}$ (inset of Fig. 3). The large softening of the $c_{\mathrm{t}}$ mode in the paramagnetic state therefore signals the importance of the 1D spin-spin correlations in the spin-strain interaction.

For the analysis of our experimental observations we consider exchange-striction coupling and apply the theory proposed in Ref. [44], subsequently developed for the description of magnetoacoustic experiments in frustrated [25-27] and quasi-one-dimensional [22,23] magnetic systems. Some details can be found in the Appendix. According to this theory the magnetic field and temperature dependence of the sound velocity and attenuation are determined by the magnetization and the magnetic susceptibility. The change of the sound attenuation (similar results can be given for the sound velocity) in the framework of the exchange-striction model can be approximated by

$$
\begin{aligned}
\Delta \alpha \approx & \frac{\gamma}{\rho V v \mu^{4}\left(\gamma^{2}+\omega^{2}\right)}\left[|g(0)|^{2}\left(2 M^{2} \chi+k_{\mathrm{B}} T \chi^{2}\right)\right. \\
& \left.+h(0) \mu^{2}\left(M^{2}+k_{\mathrm{B}} T \chi\right)\right],
\end{aligned}
$$

where $\rho$ and $V$ are the density and the volume of the crystal, respectively, $\mu$ is the effective magnetic moment of the magnetic ion, $\gamma$ is the linear relaxation parameter, $\omega$ is the sound frequency, and $k_{\mathrm{B}}$ is the Boltzmann constant. $M$ and $\chi$ are the magnetization and the magnetic susceptibility, respectively. $g$ and $h$ are given by

$$
\begin{aligned}
& g=\sum_{j} e^{i \mathbf{q} \mathbf{R}_{j i}}\left(e^{i \mathbf{k} \mathbf{R}_{j i}}-1\right) \mathbf{u}_{\mathbf{k}} \frac{\partial J_{i j}^{\beta, \beta^{\prime}}}{\partial \mathbf{R}_{i}}, \\
& h=\sum_{j} e^{i \mathbf{q} \mathbf{R}_{j i}}\left[1-\cos \left(\mathbf{k} \mathbf{R}_{j i}\right)\right]\left(\mathbf{u}_{\mathbf{k}} \cdot \mathbf{u}_{-\mathbf{k}}\right) \frac{\partial^{2} J_{i j}^{\beta, \beta^{\prime}}}{\partial \mathbf{R}_{i} \partial \mathbf{R}_{j}}
\end{aligned}
$$

(taken at $\mathbf{q}=0$ ), where $\mathbf{R}_{j i}=\mathbf{R}_{j}-\mathbf{R}_{i} ; \mathbf{R}_{j}$ is the position vector of the $j$ th site of the magnetic ion. $J_{i j}^{\beta, \beta^{\prime}}\left(\beta, \beta^{\prime}=\right.$ $x, y, z)$ are the exchange couplings between magnetic ions situated on the $i$ th and $j$ th site. While $h$ and $g$ are tensors, only single relevant components (related to the direction of the magnetic field) are used in our estimates. On the other hand, the spin-nematic contribution to the sound attenuation (a similar expression can be derived for the sound velocity) can be written as

$$
\begin{aligned}
\Delta \alpha_{\mathrm{sn}} \approx & \frac{\gamma}{\rho V v\left(\gamma^{2}+\omega^{2}\right)}\left[\left|g_{x y}(0)\right|^{2}|Q|^{2}+h_{x y}|Q|\right. \\
& \left.+k_{\mathrm{B}} T \sum_{q}\left|g_{x y}\right|^{2} \chi_{Q}(q)\right],
\end{aligned}
$$

where $Q=\left\langle S_{\mathbf{R}_{i}}^{x} S_{\mathbf{R}_{j}}^{x}-S_{\mathbf{R}_{i}}^{y} S_{\mathbf{R}_{j}}^{y}\right\rangle \exp \left(i \mathbf{q} \mathbf{R}_{j i}\right)$ is the spin-nematic order parameter (the component of the quadrupole moment), and $\chi_{Q}(q)$ is the quadrupolar susceptibility. The largest contribution comes from the $x$ and $y$ components of the exchange couplings in $g$ and $h$. Spin-nematic ordering contributes mostly to the transverse-sound properties, in accordance with our experimental findings.

The field and temperature dependence of the magnetization and the magnetic susceptibility were determined as follows. First, we calculated the magnetic moment and the magnetic susceptibility of the $1 \mathrm{D}$ spin subsystem of $\mathrm{LiCuVO}_{4}$ in the framework of the spin- $1 / 2$ chain model with NN FM and NNN AFM exchange interactions [45]. The spin-nematic contribution from a single spin chain has been estimated using results for the condensation of two-magnon bound states $[10,11,13,46]$, the spinon approach [47], and bosonization [41]; all these methods yield similar contributions to the acoustic properties. Notice that quadrupoles are not directly coupled to the magnetic field. Then, to take into account the interchain interaction, we apply the standard random-phase approximation $[48,49]$, also modified for the spin-nematic contribution. The known parameters for the in-chain NN FM exchange, NNN AFM exchange, and the interchain exchange interactions were used together with a weak magnetic anisotropy. Following Ref. [44], $\left[|g(0)|^{2} v / \rho V \mu^{4} \omega^{2}\right] \approx$ $0.323 \mathrm{~K}^{-1},\left[\mu^{2} h(0) /|g(0)|^{2}\right] \approx 0.01 \mathrm{~K}$ were used as fit parameters; the value $\gamma / v\left[1+(\gamma / \omega)^{2}\right]=|\Delta \alpha /(\Delta v / v)|$ can be obtained from the experimental results [Figs. 2(a) and 2(b)]. From the value $\mu^{2} h(0) /|g(0)|^{2}$ we can conclude that the single-phonon processes contribute more strongly to the magnetoacoustic properties than the two-phonon processes.

The result for the calculated sound attenuation of the $c_{\mathrm{t}}$ mode is presented for $T=1.5$ and $4.2 \mathrm{~K}$ by the cyan solid lines in Fig. 2(b). Spin-nematic ordering contributes mostly in the region $H_{\mathrm{c} 3} \leqslant H \leqslant H_{\text {sat }}$. This contribution decays with 
increasing temperature. Theoretically, the transverse component of the dipole moment tends to vanish in the spin-nematic phase between $H_{\mathrm{c} 3}$ and $H_{\mathrm{sat}}$, whereas the quadrupole moment is considered to become dominant [10]. The SDW phase is predicted to be the nematic-dominant Tomonaga-Luttinger liquid as approaching $H_{\mathrm{c} 3}$ [41], leading to a reduction of the dipole moments. It is obvious that close to the saturation field the magnetic moments predominately align along the magnetic field, resulting in small values of the transverse components of the magnetic moment. Hence, the longitudinal spin-dipolar component might be still important at such high fields, because the quadrupolar components are built on the basis of the relatively small transverse two-spin correlators; see the Appendix.

Our calculations reproduce the main features in the sound attenuation of $\mathrm{LiCuVO}_{4}$, foremost the two maxima at $H_{\mathrm{c} 3}$ and $H_{\text {sat }}$ [Fig. 2(b)]. The calculated temperature dependence of the sound velocity for the $c_{\mathrm{t}}$ mode at zero field (determined by the temperature dependence of the magnetic susceptibility) shows a softening with one minimum; see the Appendix. The two-minimum structure observed in the experiment (Fig. 3) is caused by additional relaxation processes that are beyond the framework of the theoretical approach. The observed sound-velocity increase in the spin-nematic phase and the hysteresis in the acoustic properties cannot be reproduced in our model [50]. Nevertheless, our calculations clearly support the application of the weakly coupled spin- $1 / 2$ chain model with NN FM and NNN AFM interactions to $\mathrm{LiCuVO}_{4}$, for which the spin-nematic phase was predicted.

\section{SUMMARY}

In summary, the observed MCE in $\mathrm{LiCuVO}_{4}$ and the derived magnetic Grüneisen parameter evidence quantum criticality $\mu_{0} H_{\mathrm{c} 3} \approx 40 \mathrm{~T}(H \| c)$ with a strong entropy increase which we attribute to the transition into the spin-nematic state occurring about $5 \mathrm{~T}$ below full magnetic saturation at $\mu_{0} H_{\mathrm{sat}} \approx 44.5 \mathrm{~T}$. The field-induced temperature change exhibits a minimum at the transition to the nematic phase with a large hysteresis in the ordered state. A large hysteresis is also detected in the acoustic properties. These are characterized by two sharp maxima at the transitions to the spinnematic and the saturated state. We can successfully model the peak structure in the attenuation of the transverse acoustic modes by a theory taking into consideration both spinstrain and quadrupole-strain interactions in the spin-nematic phase.

\section{ACKNOWLEDGMENTS}

We acknowledge support of the HLD at HZDR, member of the European Magnetic Field Laboratory (EMFL), the DFG through SFB 1143 and the excellence cluster ct.qmat (EXC 2147, Project ID 39085490), and the BMBF via DAAD (Project ID 57457940). V.O.C. acknowledges support from the Volkswagen Stiftung via Grant No. 151110. M.S.H. acknowledges the Czech Science Foundation through Project No. 19-07931Y.

\section{APPENDIX}

Here, we present the calculations used for the estimation of the contribution of the spin-nematic ordering to the sound attenuation in $\mathrm{LiCuVO}_{4}$. Results of the sound-velocity calculations at zero magnetic field are also shown. The Hamiltonian of a spin- $1 / 2$ system coupled to phonons can be written as

$$
\mathcal{H}=\mathcal{H}_{s}+\mathcal{H}_{\mathrm{ph}}+\mathcal{H}_{s-\mathrm{ph}} .
$$

Here, the spin part is

$$
\mathcal{H}_{s}=-\sum_{i, j} \sum_{\beta, \beta^{\prime}} J_{i j}^{\beta, \beta^{\prime}} S_{i}^{\beta} S_{j}^{\beta^{\prime}},
$$

with the exchange integrals $J_{i j}^{\beta \beta^{\prime}}\left(\beta, \beta^{\prime}=x, y, z\right)$, and $S_{j}^{\beta}$ being the operator of the $\beta$ projection of the spin- $1 / 2$ in the site with the coordinate $R_{j}$. The phonon part can be written as

$$
\mathcal{H}_{\mathrm{ph}}=\hbar \sum_{\mathbf{k}} \omega_{\mathbf{k}}^{0} b_{\mathbf{k}}^{\dagger} b_{\mathbf{k}}
$$

where $\hbar \omega_{\mathbf{k}}^{0}$ is the energy of the phonon mode with the wave vector $\mathbf{k}$, with $b_{\mathbf{k}}^{\dagger}\left(b_{\mathbf{k}}\right)$ being the operators of the creation (destruction) of the phonon. The spin-phonon part of the Hamiltonian is written taking into account that the exchange coupling between spins depends on the distance between the spins and the orientation of the spin-spin coupling with respect to the crystal axes and on the polarizations of phonons. It can be written as

$$
\begin{aligned}
\mathcal{H}_{\mathrm{s}-\mathrm{ph}}= & \left(\frac{\hbar}{2 \rho V}\right)^{1 / 2} \sum_{\mathbf{k}}\left[\frac{1}{\sqrt{\omega_{\mathbf{k}}^{0}}} U_{\mathbf{k}}^{(1)}\left(b_{\mathbf{k}}^{\dagger}+b_{\mathbf{k}}\right)\right. \\
& \left.+\sum_{\mathbf{k}^{\prime}} \frac{U_{k, k^{\prime}}^{(2)}}{\sqrt{\omega_{\mathbf{k}^{\prime}}^{0} \omega_{\mathbf{k}}^{0}}}\left(b_{\mathbf{k}^{\prime}}^{\dagger} b_{\mathbf{k}}+b_{-\mathbf{k}}^{\dagger} b_{-\mathbf{k}^{\prime}}+b_{\mathbf{k}} b_{-\mathbf{k}^{\prime}}+b_{-\mathbf{k}^{\prime}}^{\dagger} b_{\mathbf{k}}^{\dagger}\right)\right],
\end{aligned}
$$

with

$$
\begin{aligned}
U_{\mathbf{k}}^{(1)}= & -\left(e^{i \mathbf{k} \mathbf{R}_{j i}}-1\right) \mathbf{u}_{\mathbf{k}} \sum_{\beta, \beta^{\prime}} \frac{\partial J_{i j}^{\beta, \beta^{\prime}}}{\partial \mathbf{R}_{i}} S_{i}^{\beta} S_{j}^{\beta^{\prime}}, \\
U_{\mathbf{k}, \mathbf{k}^{\prime}}^{(2)}= & -\frac{1}{2} \sum_{j} e^{i \mathbf{q} \mathbf{R}_{j i}}\left[1-\cos \left(\mathbf{k} \mathbf{R}_{j i}\right)\right] \\
& \times\left(\mathbf{u}_{\mathbf{k}} \cdot \mathbf{u}_{-\mathbf{k}}\right) \sum_{\beta, \beta^{\prime}} \frac{\partial^{2} J_{i j}^{\beta, \beta^{\prime}}}{\partial \mathbf{R}_{i} \partial \mathbf{R}_{j}} S_{i}^{\beta} S_{j}^{\beta^{\prime}} .
\end{aligned}
$$

One can see that two terms in the spin-phonon Hamiltonian $\mathcal{H}_{s \text {-ph }}$ describe the single-phonon and two-phonon processes, which yield terms proportional to the coefficients $g$ and $h$ in the main text.

Let us define the relaxation function of any two operators $A$ and $B$ as

$$
(A, B) \equiv \int_{0}^{1 / k_{\mathrm{B}} T} d x\left\langle e^{x \mathcal{H}} A e^{-x \mathcal{H}}, B\right\rangle-\frac{\langle A\rangle\langle B\rangle}{k_{\mathrm{B}} T},
$$

where $k_{\mathrm{B}}$ is the Boltzmann constant, $T$ is the temperature, and

$$
\langle A\rangle=\frac{\operatorname{Tr} A e^{-\mathcal{H} / k_{\mathrm{B}} T}}{\operatorname{Tr} e^{-\mathcal{H} / k_{\mathrm{B}} T}} .
$$


Let us define also the random force

$$
f_{\mathbf{k}}=\dot{b}_{\mathbf{k}}+i \omega_{\mathbf{k}} b_{\mathbf{k}},
$$

where the dot determines the time derivative. Then, according to Ref. [51], the attenuation coefficient for the phonon can be written as

$$
\Delta \alpha_{\mathbf{k}}=\operatorname{Re} \int_{0}^{\infty} d t e^{-i \omega_{\mathbf{k}} t} \frac{f_{\mathbf{k}}(t) f_{\mathbf{k}}^{\dagger}(0)}{v\left(b_{\mathbf{k}}, b_{\mathbf{k}}^{\dagger}\right)},
$$

where $v$ is the velocity of the sound wave. Using the relation

$$
\hbar \omega_{\mathbf{k}}=i \hbar \frac{\left(\dot{b}_{\mathbf{k}}, b_{\mathbf{k}}^{\dagger}\right)}{\left(b_{\mathbf{k}}, b_{\mathbf{k}}^{\dagger}\right)}=\frac{1}{\left(b_{\mathbf{k}}, b_{\mathbf{k}}^{\dagger}\right)},
$$

the denominator in the equation for $\alpha_{\mathbf{k}}$ can be written as $\hbar \omega_{\mathbf{k}}$. Since the changes in $v$ and $\omega_{\mathbf{k}}$ are small for weak spin-phonon coupling, we can approximate them by the ones for the noninteracting phonon system.

Consider the effect of the single-phonon term of the spinphonon Hamiltonian (the term for the two-phonon coupling can be considered in an analogous way, however, with more evolved calculations). The random force can be written as

$$
f_{\mathbf{k}}=-\frac{i U_{-\mathbf{k}}^{(1)}}{\sqrt{2 \rho V \hbar \omega_{\mathbf{k}}^{0}}} .
$$

Then, the attenuation coefficient can be written as

$$
\begin{aligned}
\Delta \alpha_{\mathbf{k}}= & \frac{1}{2 \rho V v} \operatorname{Re} \int_{0}^{\infty} d t e^{-i \omega_{\mathbf{k}} t} \sum_{\mathbf{q}, \mathbf{q}^{\prime}} \sum_{\beta, \beta^{\prime}}\left(g_{\mathbf{q}}\right)^{*} g_{\mathbf{q}^{\prime}} \\
& \times\left(S_{\mathbf{q}}^{\beta}(t) S_{-\mathbf{q}-\mathbf{k}}^{\beta}(t), S_{-\mathbf{q}^{\prime}}^{\beta^{\prime}}(0) S_{\mathbf{q}^{\prime}+\mathbf{k}}^{\beta^{\prime}}(0)\right),
\end{aligned}
$$

where we introduced the Fourier transformations of the operators of the spin projections, and the coefficients $g$ are determined in the main text. A similar expression, proportional to $h$, can be written for the contribution from the two-phonon term, taking into account the weak spin-phonon coupling. We can see that the sound attenuation is proportional to a four-spin correlation function.

Usually the magnetic (dipole) degrees of freedom are considered, and the four-spin correlation function can be replaced by the combination of the magnetization and magnetic susceptibility [44]. Such an approach yields Eqs. (1) and (2).

However, here it is necessary to take into account the possibility of spin-nematic ordering, with $Q=\left\langle S_{\mathbf{R}_{i}}^{x} S_{\mathbf{R}_{j}}^{x}-\right.$ $\left.S_{\mathbf{R}_{i}}^{y} S_{\mathbf{R}_{j}}^{y}\right\rangle \exp \left(i \mathbf{q} \mathbf{R}_{j i}\right)$ being the spin-nematic order parameter (the component of the quadrupole moment). Namely, such contribution is expected for $\mathrm{LiCuVO}_{4}$ [10]. We also assume that the wave vector of the sound is small. In the decoupling approximation one can write for any four operators $A, B, C, D$ the following relation:

$$
\begin{aligned}
(A B, C D) \approx & k_{\mathrm{B}} T[(A, B)(C, D)+(A, C)(B, D) \\
& +(A, D)(B, C)]-\langle A B\rangle\langle C D\rangle .
\end{aligned}
$$

We use this approximation for the Fourier components of the operators of the projections of the spins. Then, we write any two-spin operator for $\beta, \beta^{\prime}=x, y$ as $S_{i}^{\beta} S_{j}^{\beta^{\prime}}=Q+\left(S_{i}^{\beta}\right)^{\prime}\left(S_{j}^{\beta^{\prime}}\right)^{\prime}$, with the second term describing the deviation from the order parameter. Obviously, the four-spin correlation function for the deviations can be written via the quadrupolar susceptibility. Supposing homogeneous damping for each spin operator,

$$
\left(S_{\mathbf{q}}^{\beta}(t) S_{-\mathbf{q}}^{\beta}(0)\right) \approx\left(S_{\mathbf{q}}^{\beta} S_{-\mathbf{q}}^{\beta}\right) e^{-\gamma t},
$$

where $\gamma$ is the damping coefficient, we can write the final approximated expression for the contribution of the spin-nematic ordering to the sound attenuation (up to logarithmic corrections)

$$
\begin{aligned}
\Delta \alpha_{\mathbf{k}} \approx & \frac{\gamma}{\rho V v\left[\gamma^{2}+\left(\omega_{\mathbf{k}}^{0}\right)^{2}\right]}\left[\left|g_{x y}(0)\right|^{2}|Q|^{2}+h_{x y}|Q|\right. \\
& \left.+k_{\mathrm{B}} T \sum_{q}\left|g_{x y}\right|^{2} \chi_{Q}(q)\right]
\end{aligned}
$$

where $\chi_{Q}(q)$ is the quadrupolar susceptibility. For our estimations we can also approximate $\omega_{\mathbf{k}}^{0}$ by the cyclic frequency of the sound.

Now we have to calculate $Q$ and $\chi_{Q}(q)$. We can do this, e.g., using the bosonization approach [41]. The quadrupolar susceptibility of the spin chain is maximal at $q=\pi$. It can be written as

$$
\begin{aligned}
\chi_{Q}(q=\pi) \approx & \frac{1}{v_{\mathrm{F}}}\left(\frac{4 \pi k_{\mathrm{B}} T}{v_{\mathrm{F}}}\right)^{2 / K-2} \\
& \times|\sin (\pi / K)| B^{2}\left(\frac{1}{2 K}, 1-\frac{1}{K}\right),
\end{aligned}
$$

where $v_{\mathrm{F}}$ is the Fermi velocity, $K$ is the Luttinger-liquid exponent, and $B(x, y)$ is the Beta function. In general, these parameters depend on $H$. For $H=H_{\text {sat }}$ the velocity is zero [41], and $K=1$. On the other hand, for $H=0$ we have $K=1 / 2$. The quadrupolar susceptibility of the quasi-1D system in the random-phase approximation can be written as

$$
\chi_{3} \approx \frac{\chi_{Q}(q=\pi)}{1-z J_{\text {eff }} \chi_{Q}(q=\pi)},
$$

where $z$ is the coordination number and $J_{\text {eff }}$ is the effective coupling between the spin chains. For our purposes, we can approximate $J_{\text {eff }}$ by $J$ (see the main text for the value of $J$ ). The spin-nematic order parameter $Q$ in the ground state can be written as [11]

$$
|Q|=\sqrt{\frac{v_{\mathrm{F}}}{2 \pi D}}
$$

where

$$
D=\left|J_{1}\right| \frac{3 x^{2}(2+x)-1}{16(1+x)^{2}},
$$

with $x=\left|J_{2} / J_{1}\right|$ (see the main text for the values of $J_{1}$ and $J_{2}$ ). Finally, we can adopt the approximate expression for $v_{\mathrm{F}}(H)$ and $K(H)$ from Refs. [49,52]. Namely, we can write

$$
v_{\mathrm{F}}=\frac{\pi J_{2}}{2} \sqrt{\left(1-\frac{H}{H_{\mathrm{sat}}}\right)\left(1-\frac{H}{H_{\mathrm{sat}}}+\frac{2 \mu H}{\pi J_{2}}\right)},
$$




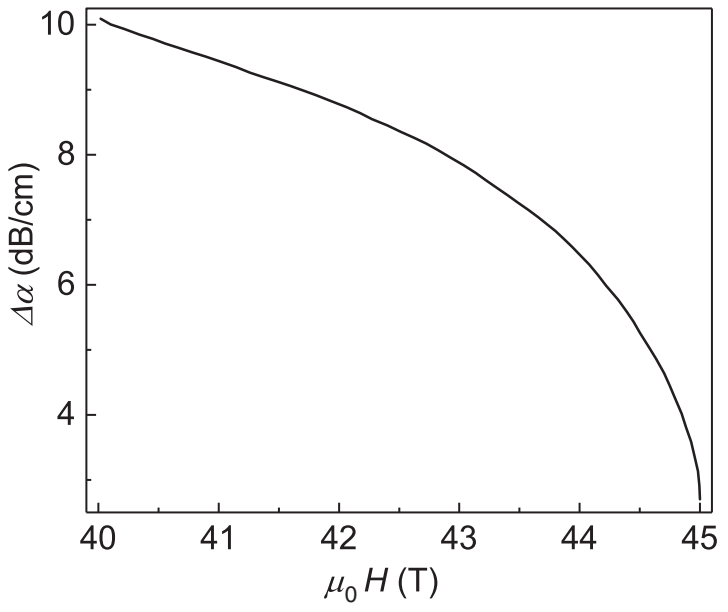

FIG. 4. Field dependence of the spin-quadrupolar contribution to the sound attenuation $\Delta \alpha$ of the transverse $c_{\mathrm{t}}$ mode at $T=1.5 \mathrm{~K}$ for the quasi-1D spin- $1 / 2$ chain model.

and

$$
\begin{aligned}
& K=\frac{f}{\sqrt{4 f^{2}-3(\mu H)^{2}}}, \\
& f=\mu H+\pi J_{2}\left(1-\frac{H}{H_{\text {sat }}}\right) .
\end{aligned}
$$

Figure 4 shows the field dependence of the quadrupolar contribution to the sound attenuation in the field range $H_{\mathrm{c} 3}<$ $H<H_{\text {sat }}$ at $T=1.5 \mathrm{~K}$ (we remind the reader that the order parameter $Q \neq 0$ only in this field region, and in our calculations we used the order-parameter ground-state value; the nonzero temperature yields the decay of the order parameter). We used the value for the parameter $\gamma / v\left[1+(\gamma / \omega)^{2}\right]$, as for the calculation of the sound attenuation in Fig. 2 of the main text. By comparing the attenuation change from Fig. 2(b) in the main text with Fig. 4, we see that the contribution from the spin-nematic ordering is about $10 \%$ of the contribution from the magnetic dipoles. Alternatively, we have also estimated the values of $Q$ and $\chi_{Q}$ using the spin-wave approach of Ref. [11] (which gives similar results to Refs. [10,46]). Such an estimation yields even smaller contributions from the spinnematic parameters than the one presented in Fig. 4.

This relatively small contribution is understood in terms of the two-spin correlators. As we have mentioned in the main

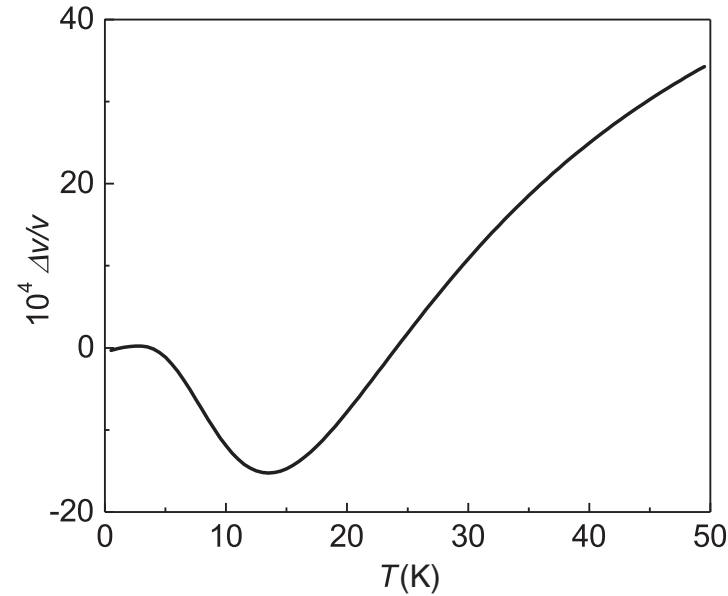

FIG. 5. Temperature dependence of the sound velocity of the transverse $c_{\mathrm{t}}$ mode at zero magnetic field for the quasi-1D spin-1/2 chain model.

text, close to the saturation field, in the spin-nematic phase, the magnetic moments predominately align along the magnetic field, leading to small values of the two-spin correlators for the transverse components of the magnetic moment from which the quadrupolar components are built. Hence, the dipoles might still dominate at such high magnetic fields. This might be the reason for the relatively small quadrupolar contribution. Moreover, we assumed that the damping of the dipolar and quadrupolar parts are the same. This is not necessarily the case. Changing the ratio between these dampings would significantly change the value of the quadrupolar contribution (while the field dependence would remain similar). The spinnematic contribution, decreasing with field between $H_{\mathrm{c} 3}$ and $H_{\text {sat }}$, leads to the partial suppression of the dipole-defined sound attenuation.

We calculated the sound velocity at zero field using a relation similar to one given in the main text for the sound attenuation within the exchange-striction model $\Delta v / v=f(E-$ $k_{\mathrm{B}} T C_{p}$ ), where $f \approx 7 \times 10^{-4} \mathrm{~K}^{-1}$ is the fit parameter, $E$ is the internal energy, and $C_{p}$ is the heat capacity $[53,54]$. Figure 5 shows the calculated temperature dependence of the sound velocity for the $c_{\mathrm{t}}$ mode at zero field (determined by the temperature dependence of the magnetic susceptibility of the quasi-1D spin chain). The characteristic softening of the acoustic mode, similar to one observed in the experiment, appears due to the strong 1D spin-spin correlations.
[1] A. F. Andreev and I. A. Grishchuk, Zh. Eksp. Teor. Fiz. 87, 467 (1984) [Sov. Phys. JETP 60, 267 (1984)].

[2] A. V. Prokofiev, I. G. Vasilyeva, V. N. Ikorskii, V. V. Malakhov, I. P. Asanov, and W. Assmus, J. Solid State Chem. 177, 3131 (2004).

[3] M. Enderle, C. Mukherjee, B. Fåk, R. K. Kremer, J.-M. Broto, H. Rosner, S.-L. Drechsler, J. Richter, J. Malek, A. Prokofiev, W. Assmus, S. Pujol, J.-L. Raggazzoni, H. Rakoto, M. Rheinstädter, and H. M. Rønnow, Europhys. Lett. 70, 237 (2005).
[4] B. J. Gibson, R. K. Kremer, A. V. Prokofiev, W. Assmus, and G. J. McIntyre, Phys. B (Amsterdam) 350, e253 (2004).

[5] F. Heidrich-Meisner, I. P. McCulloch, and A. K. Kolezhuk, Phys. Rev. B 80, 144417 (2009).

[6] M. Enderle, B. Fåk, H.-J. Mikeska, R. K. Kremer, A. Prokofiev, and W. Assmus, Phys. Rev. Lett. 104, 237207 (2010).

[7] N. Büttgen, W. Kraetschmer, L. E. Svistov, L. A. Prozorova, and A. Prokofiev, Phys. Rev. B 81, 052403 (2010).

[8] N. Büttgen, P. Kuhns, A. Prokofiev, A. P. Reyes, and L. E. Svistov, Phys. Rev. B 85, 214421 (2012). 
[9] T. Masuda, M. Hagihala, Y. Kondoh, K. Kaneko, and N. Metoki, J. Phys. Soc. Jpn. 80, 113705 (2011).

[10] M. E. Zhitomirsky and H. Tsunetsugu, Europhys. Lett. 92, 37001 (2010).

[11] A. V. Syromyatnikov, Phys. Rev. B 86, 014423 (2012).

[12] N. Shannon, T. Momoi, and P. Sindzingre, Phys. Rev. Lett. 96, 027213 (2006).

[13] H. T. Ueda and T. Momoi, Phys. Rev. B 87, 144417 (2013).

[14] A. Smerald and N. Shannon, Phys. Rev. B 88, 184430 (2013).

[15] O. A. Starykh, Rep. Prog. Phys. 78, 052502 (2015).

[16] L. E. Svistov, T. Fujita, H. Yamaguchi, S. Kimura, K. Omura, A. Prokofiev, A. I. Smirnov, Z. Honda, and M. Hagiwara, JETP Lett. 93, 21 (2011).

[17] A. Orlova, E. L. Green, J. M. Law, D. I. Gorbunov, G. Chanda, S. Krämer, M. Horvatić, R. K. Kremer, J. Wosnitza, and G. L. J. A. Rikken, Phys. Rev. Lett. 118, 247201 (2017).

[18] N. Büttgen, K. Nawa, T. Fujita, M. Hagiwara, P. Kuhns, A. Prokofiev, A. P. Reyes, L. E. Svistov, K. Yoshimura, and M. Takigawa, Phys. Rev. B 90, 134401 (2014).

[19] Z. Wang, T. Lorenz, D. I. Gorbunov, P. T. Cong, Y. Kohama, S. Niesen, O. Breunig, J. Engelmayer, A. Herman, J. Wu, K. Kindo, J. Wosnitza, S. Zherlitsyn, and A. Loidl, Phys. Rev. Lett. 120, 207205 (2018).

[20] M. Garst and A. Rosch, Phys. Rev. B 72, 205129 (2005).

[21] B. Lüthi, Physical Acoustics in the Solid State (Springer, Berlin, 2005).

[22] O. Chiatti, A. Sytcheva, J. Wosnitza, S. Zherlitsyn, A. A. Zvyagin, V. S. Zapf, M. Jaime, and A. Paduan-Filho, Phys. Rev. B 78, 094406 (2008).

[23] A. Sytcheva, O. Chiatti, J. Wosnitza, S. Zherlitsyn, A. A. Zvyagin, R. Coldea, and Z. Tylczynski, Phys. Rev. B 80, 224414 (2009).

[24] J. Wosnitza, S. A. Zvyagin, and S. Zherlitsyn, Rep. Prog. Phys. 79, 074504 (2016).

[25] S. Erfanifam, S. Zherlitsyn, J. Wosnitza, R. Moessner, O. A. Petrenko, G. Balakrishnan, and A. A. Zvyagin, Phys. Rev. B 84, 220404(R) (2011).

[26] S. Erfanifam, S. Zherlitsyn, S. Yasin, Y. Skourski, J. Wosnitza, A. A. Zvyagin, P. McClarty, R. Moessner, G. Balakrishnan, and O. A. Petrenko, Phys. Rev. B 90, 064409 (2014).

[27] S. Bhattacharjee, S. Erfanifam, E. L. Green, M. Naumann, Z. Wang, S. Granovsky, M. Doerr, J. Wosnitza, A. A. Zvyagin, R. Moessner, A. Maljuk, S. Wurmehl, B. Büchner, and S. Zherlitsyn, Phys. Rev. B 93, 144412 (2016).

[28] M. Mourigal, M. Enderle, B. Fåk, R. K. Kremer, J. M. Law, A. Schneidewind, A. Hiess, and A. Prokofiev, Phys. Rev. Lett. 109, 027203 (2012).

[29] A. V. Prokofiev, D. Wichert, and W. Assmus, J. Cryst. Growth 220, 345 (2000).

[30] Ch. Kegler, N. Büttgen, H.-A. Krug von Nidda, A. Krimmel, L. Svistov, B. I. Kochelaev, A. Loidl, A. Prokofiev, and W. Assmus, Eur. Phys. J. B 22, 321 (2001).

[31] M. A. Lafontaine, M. Leblanc, and G. Ferey, Acta Cryst. C 45, 1205 (1989).

[32] M. Li, X. Yang, C. Wang, N. Chen, F. Hu, X. Bie, Y. Wei, F. Du, and G. Chen, J. Mater. Chem. A 3, 586 (2015).
[33] T. Kihara, Y. Kohama, Y. Hashimoto, S. Katsumoto, and M. Tokunaga, Rev. Sci. Instrum. 84, 074901 (2013).

[34] M. G. Banks, F. Heidrich-Meisner, A. Honecker, H. Rakoto, J.-M. Broto, and R. K. Kremer, J. Phys.: Condens. Matter 19, 145227 (2007).

[35] O. A. Starykh and L. Balents, Phys. Rev. B 89, 104407 (2014).

[36] L. A. Prozorova, S. S. Sosin, L. E. Svistov, N. Büttgen, J. B. Kemper, A. P. Reyes, S. Riggs, A. Prokofiev, and O. A. Petrenko, Phys. Rev. B 91, 174410 (2015).

[37] N. Büttgen, H.-A. Krug von Nidda, L. E. Svistov, L. A. Prozorova, A. Prokofiev, and W. Aßmus, Phys. Rev. B 76, 014440 (2007).

[38] O. Breunig, M. Garst, A. Klümper, J. Rohrkamp, M. M. Turnbull, and T. Lorenz, Sci. Adv. 3, eaao3773 (2017).

[39] J. Wu, L. Zhu, and Q. Si, Phys. Rev. B 97, 245127 (2018).

[40] K. Yu. Povarov, V. K. Bhartiya, Z. Yan, and A. Zheludev, Phys. Rev. B 99, 024413 (2019).

[41] M. Sato, T. Hikihara, and T. Momoi, Phys. Rev. Lett. 110, 077206 (2013).

[42] The short sample length along the $c$ direction allowed only for a resolution of $\sim 10^{-3}$ in $\Delta v / v$ for the $c_{33}$ mode in a pulsed magnetic field. This did not allow us to resolve any anomalous behavior for $c_{33}$.

[43] Y. P. Varshni, Phys. Rev. B 2, 3952 (1970).

[44] M. Tachiki and S. Maekawa, Prog. Theor. Phys. 51, 1 (1974).

[45] These results are obtained using exact diagonalization of small clusters for an even number of sites in the chain (up to 20 sites). Since it is well known that calculations for finite spin chains differ for chains with an even number of sites proportional to $2 n$ and $4 n$ ( $n$ is a positive integer), we average the obtained results with respect to such chains with lengths $2 n$ and $4 n$. Additional lower-field peaks in Fig. 2(b) are probably related to the finite-size effects. Their magnitude decays with the growth of the cluster size.

[46] S. Nishimoto, S.-L. Drechsler, R. Kuzian, J. Richter, and J. van den Brink, Phys. Rev. B 92, 214415 (2015).

[47] S.-S. Zhang, N. Kaushal, E. Dagotto, and C. D. Batista, Phys. Rev. B 96, 214408 (2017).

[48] A. A. Zvyagin and S.-L. Drechsler, Phys. Rev. B 78, 014429 (2008).

[49] A. A. Zvyagin, Phys. Rev. B 81, 224407 (2010).

[50] The discrepancy is probably related to our simplified approach, which does not take into account fluctuations of the dipolar and quadrupolar order parameters, and of the $\mathbf{q}$ dependence of the magnetic susceptibility.

[51] H. Mori, Prog. Theor. Phys. 33, 423 (1965).

[52] H. Kühne, A. A. Zvyagin, M. Günther, A. P. Reyes, P. L. Kuhns, M. M. Turnbull, C. P. Landee, and H.-H. Klauss, Phys. Rev. B 83, 100407(R) (2011).

[53] S. Bhattacharjee, S. Zherlitsyn, O. Chiatti, A. Sytcheva, J. Wosnitza, R. Moessner, M. E. Zhitomirsky, P. Lemmens, V. Tsurkan, and A. Loidl, Phys. Rev. B 83, 184421 (2011).

[54] A. V. Andreev, S. Yasin, Y. Skourski, A. A. Zvyagin, S. Zherlitsyn, and J. Wosnitza, Phys. Rev. B 87, 214409 (2013). 